\title{
Femtosecond filamentation in air and higher-order nonlinearities
}

\author{
M. Kolesik, E. M. Wright, and J.V. Moloney \\ College of Optical Sciences, University of Arizona, Tucson AZ 85721
}

Compiled May 29, 2022

\begin{abstract}
According to a recent experiment, the instantaneous electronic Kerr effect in air exhibits a strong intensity dependence, the nonlinear refractive index switching sign and crossing over from a self-focusing to a de-focusing nonlinearity. A subsequent theoretical work has demonstrated that this has paradigm-changing consequences for the understanding of filamentation in air, so it is important to subject the idea of higher-order nonlinearities to stringent tests. Here we use numerical modeling to propose an experiment capable of discriminating between the standard and the new intensity-dependent Kerr-effect models. C) 2022 Optical Society of America OCIS codes: $320.2250,320.7110$.
\end{abstract}

Over the last sesquidecade research into femtosecond filamentation in air has produced a widely accepted picture in which a dilute plasma, generated in the highintensity optical field, is a major determinant of the light pulse dynamics. For gaseous media, this scenario is based on the notion of a dynamical balance between the Kerrinduced self-focusing and the counteracting de-focusing caused by the free electrons [1. Higher-order nonlinearities have previously been considered before as an additional mechanism regularizing the self-focusing collapse. In particular, phenomenological Kerr-effect saturation at high intensities (see e.g. 2, 4] and Sec 3.4. of [5]) was explored in filament modeling. In several papers quantummechanical simulations of the electronic polarization response (e.g. [6] and references therein) were used to study the interplay of the saturated Kerr and plasma contributions. Nevertheless, in the absence of experimental evidence for these effects, the consensus in the filamentation community was that the standard self-focusing Kerr effect was sufficient to describe the electronic nonlinearity. Moreover, many experiments clearly showed the presence of plasma in filaments, and this was accepted as the mechanism clamping the intensity [7.

Recent experimental results by Loriot et al. [8,9] have the potential to change this long-standing paradigm. They found that the instantaneous Kerr nonlinearity exhibits a strong intensity dependence, and that it not only saturates, but even changes from self-focusing to de-focusing at large enough intensity. As a consequence, the plasma is not necessary for the arrest of the selffocusing collapse, because the Kerr-induced de-focusing at high intensity can alone be sufficient. Inspired by these findings, the recent modeling work of Ref. [10] proposed that contrary to our current belief, ionization-free filamentation is possible in gases.

This has been a surprising development to say the least, and one which poses many questions. The first one is of course, why the earlier experiments did not detect such a strong intensity dependence of the Kerr effect? Further, is it just an accident that the cross-over from self-focusing to de-focusing occurs in the vicinity of the intensity range where plasma starts to form, or is there a connection between the two? In particular, central to the interpretation of the Loriot's experiment is the assumption that the optical response of the plasma is isotropic, which is certainly true for the simple Drude model. However, ultrafast birefringence is know to occur for free electrons in semiconductors due to anisotropic state filling [11, and if a similar effect arises for the freecarrier plasma in air it could change the interpretation of the experiment. And perhaps the most fundamental question is what is the microscopic origin of the signchange in the Kerr effect if not due to plasma?

Since the observations of Loriot et al. have such large implications for the physical picture of filamentation in gases it is important that they be corroborated in similar experiments, and that the occurrence of higher-order nonlinearities be subjected to independent experimental testing. This may not be easy, because accurate quantitative measurements in filaments are notoriously difficult. It is also conceivable that the Kerr intensity-dependence could be partially masked by the plasma induced defocusing. In fact, this may very well be the reason why this effect has not been observed earlier. It is therefore the view of the present authors that this problem needs to be addressed with an utmost caution. Before making conclusions with regards to the filamentation dynamics, ways should be found to independently test the interpretation of the Loriot's experiment. As a first step in this direction, based on numerical simulations we propose the design of an experiment which should be capable to discriminate between the two model candidates: i.e. between the standard Kerr, and the higher-order Kerr effects.

The proposed experiment is simple in principle. One should use a modest pulse energy to generate third- and fifth-harmonic radiation, and measure their respective conversion efficiencies. To make this feasible, the fundamental frequency should be "shifted" to a longer wavelength, perhaps by using an OPA, such that the third and fifth harmonic energies can be simultaneously measured. In order to minimise the propagation effects, the focusing geometry should be tight, resulting in a short interaction volume. The aim is to compare the fifth-harmonic gen- 
eration to that of the third harmonic, and decide if the result is in keeping with the predictions of either of the competing models. The rationale behind this is the simple fact that the higher-order Kerr effect should result in a much stronger harmonic generation. Indeed, in the standard model only the third harmonic is produced directly from the fundamental, while the fifth (and higher) harmonics arise via a cascade from the third. On the other hand, the fifth harmonic can be produced directly from the fundamental in the higher-order Kerr model.

In our numerical experiments we use the Unidirectional Pulse Propagation Equation simulator 12 which works directly with the real electric field (as opposed to amplitude envelopes), and therefore naturally and without any modifications captures all frequency-mixing interactions. We assume the $50 \mathrm{fs}$ input pulse with a central wavelength of $1330 \mathrm{~nm}$ and specify its nominal intensity and beam waist which would be achieved in a linear focus. The results presented in this paper were obtained for the nominal focused beam waist of 50 microns, and the nominal in-focus intensity between $10^{17}$ and $10^{18} \mathrm{~W} / \mathrm{m}^{2}$. This tight focusing results in the interaction region only several centimeters long and is meant to suppress the possible propagation- and plasma-related effects. For the plasma generation, we use the so-called PPT ionization rates [13] parametrized as in Ref. 14. Besides the instantaneous Kerr effect, we include the usual delayed Raman component 12 with the instantaneous-to-delayed ratio of one half.

It should be emphasized that the only difference between the comparative simulation runs is the different inputs specifying the nonlinear index as a function of the instantaneous value of the electric field. For the higherorder Kerr effect model, we have (with suitable electric field units) $\Delta n=n_{2} E^{2}+n_{4} E^{4}+n_{6} E^{6}+n_{8} E^{8}$ with the $n_{2 k}$ values for air fixed according to [9]. The standard model shares the same $n_{2}$ and has $n_{4}=n_{6}=n_{8}=0$.

Let us first look at the typical spectra generated by the two models. Figure 1 shows the results for the nominal focal intensity of $10^{18} \mathrm{~W} / \mathrm{m}^{2}$ (the actual intensity reached in the interaction volume is somewhat smaller). As expected, we can see a drastic difference. First, in the new model the third-harmonic generation is almost two orders of magnitude stronger. Second, the fifth harmonic generation is four to six orders of magnitude stronger. Thus, if the new model is correct the fifth harmonic should be easily detected.

To quantify the above observation further, in Fig. 2 we show how the third- and fifth-harmonic conversion efficiencies evolve with the increasing pulse energy (or, equivalently, with the nominal focal intensity). The standard model produces the $3 \mathrm{H}$ efficiency approaching fraction of a percent which is in agreement with both experiments (see e.g [15, 16]) and previous simulations [17. On the other hand, the $3 \mathrm{H}$ efficiency in the higher-order model can be as high as several percent! To our best knowledge this has not been observed yet.

However, one could argue that a mere order of mag-

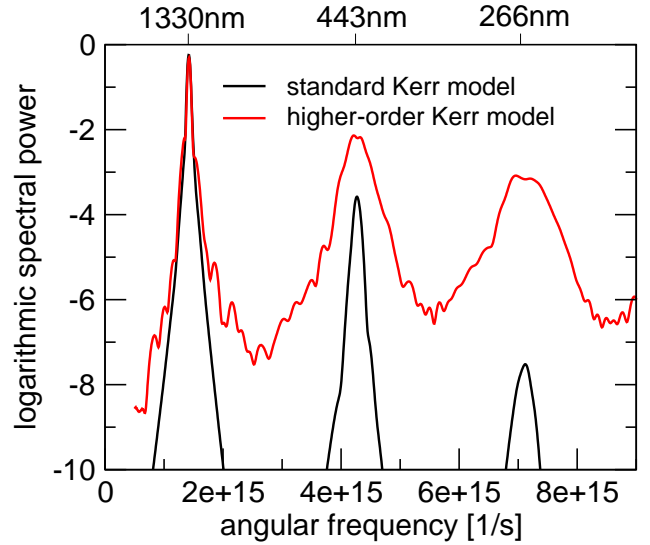

Fig. 1. Angle-integrated spectrum showing third- and fifth-harmonic generation. The standard and higherorder Kerr effect models are compared for a tightly focused femtosecond pulse. The new model predicts stronger third-harmonic generation and several orders of magnitude more powerful fifth-harmonic radiation.

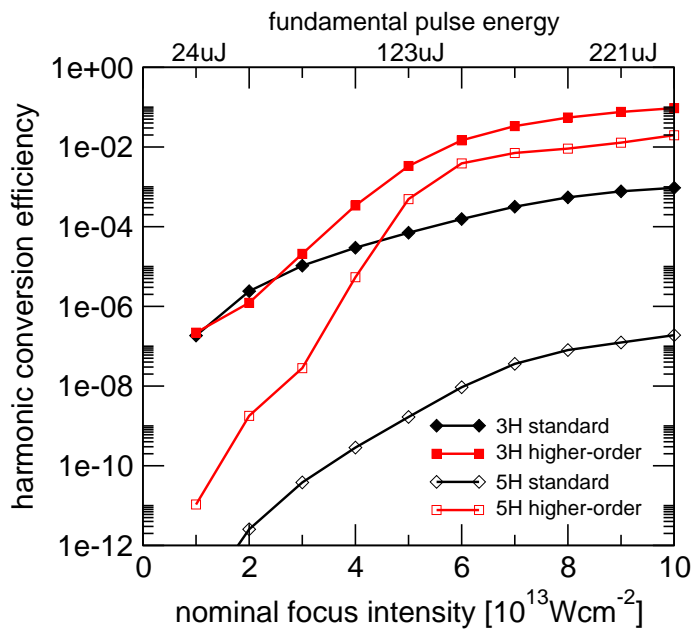

Fig. 2. The harmonic conversion efficiency as a function of nominal focal intensity/energy. Full and open symbols represent the third and fifth harmonics, and squares and diamonds represent the higher-order and standard model, respectively.

nitude difference in the third-harmonic conversion efficiency might be difficult to detect experimentally, because any real experiment will only probe "a single model version." That is why we now turn to the fifth harmonic conversion efficiency. Figure 2 shows that here the difference reaches five orders of magnitude, and this should indeed be possible to identify in the experiment. In fact, if the standard model is correct, the fifth harmonic might be hardly detectable as it should be seven orders of magnitude weaker than the fundamental. If on the other hand the new model is correct, the fifth harmonic should be easily detected on the level of up to a few percent of the fundamental energy.

Another potentially useful telltale sign which an ex- 


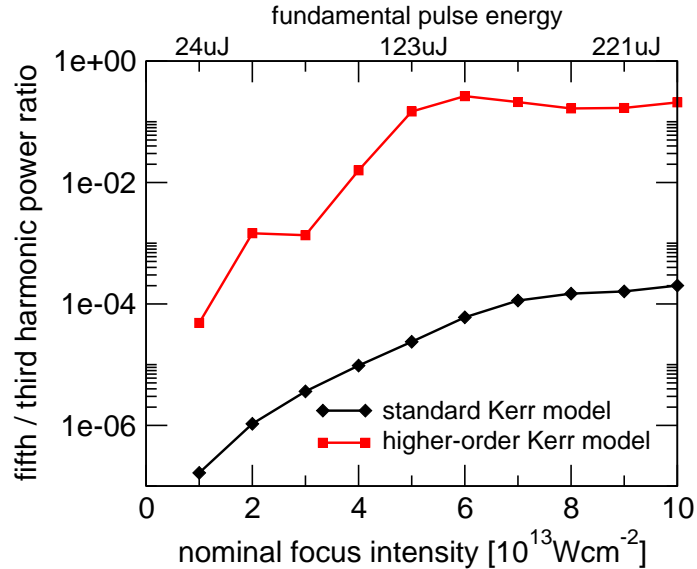

Fig. 3. Ratio of the fifth- to the third-harmonic energy generated in the tightly focused femtosecond pulse. The standard model ratio roughly corresponds to an exponential decrease between the harmonic orders. In contrast, the new model predicts that the energy carried by the fifth harmonic can be a mere order of magnitude less or even comparable to that in the third harmonic.

periment may look at is the ratio between the fifth and third harmonic production. This is shown in Fig 3 , While the standard model exhibits a small ratio reflecting the fact that the process is a cascade and the fifth harmonic production feeds on the third harmonic energy, the two harmonics become almost comparable in strength in the new model. This occurs as soon as the focal intensities reach values beyond the turn-over point of the higherorder Kerr effect. In fact, it is easy to envision that once the electric field amplitude increases beyond the turnover point, the nonlinear index change will exhibit two peaks within each half-cycle of the driving field. This in turn means that the nonlinear index spectrum contains roughly the same amounts of second and fourth harmonics giving rise to comparable strengths of the third and fifth harmonic components in the nonlinear polarization. In a nutshell, this is the reason why the new higherorder Kerr model should produce extremely strong fifthharmonic radiation.

Let us summarize our findings. Already the present calculations suggest that the standard model is in a better agreement with previous experiments on the thirdharmonic generation. It exhibits the conversion efficiencies comparable with the measured values. In contrast, the new model predicts significantly stronger third harmonic, with its energy reaching up to several percent of the fundamental. To our knowledge such a strong thirdharmonic generation has not been observed. However, we should keep in mind that due to the model parameter uncertainties the possibility can not be excluded that the simulated efficiency value may suffer from a systematic deviation.

That is why we propose an experiment which should compare the fifth and third harmonic generation. We have shown that if the higher-order Kerr effect model is correct, the experiment will detect significant fifthharmonic generation. Moreover, at higher pulse energies the relative strength of the fifth to the third harmonic should reach values of the order of $10^{-1}$. On the other hand, if the standard model is correct, this ratio should be about four to five orders smaller. This is a significant qualitative difference, and it should be obvious from the experimental results which of the competing models should be preferred.

In our view, the importance of the independent corroboration of the experiment by Loriot et al. can not be overemphasized. Their results imply literally a change of paradigm in our understanding of femtosecond filamentation, put in doubt concepts such as plasma-mediated intensity clamping, and essentially invalidate the very model of femtosecond filamentation that has been used for over a decade. It is thus our aim and hope that this work will motivate and inspire experimental efforts to solve this exciting and important problem.

This work was supported by Air Force Office of Scientific Research under contract FA9550-07-1-0010.

\section{References}

1. A. Couairon and A. Mysyrowicz, Phys. Rep. 441, 47 (2007).

2. N. Aközbek, M. Scalora, C. M. Bowden, and S. L. Chin, Opt. Commun. 191, 353 (2001).

3. A. Couairon, Phys. Rev. A 68, 015801 (2003).

4. A. Vincotte and L. Berge, Phys. Rev. A 70, 061802R (2004).

5. L. Berge, S. Skupin, R. Nuter, J. Kasparian, and J. Wolf, Rep. Prog. Phys. 71 (2008).

6. K. M. M. Nurhuda, A. Suda, New J. Phys. 10, 053006 (2008).

7. Y. P. Deng, J. B. Zhu, Z. G. Ji, J. S. Liu, B. Shuai, R. X. Li, Z. Z. Xu, F. Thberge, and S. L. Chin, Opt. Letters 31, 546 (2006).

8. V. Loriot, E. Hertz, O. Faucher, and B. Lavorel, Opt. Express 17, 13429 (2009).

9. V. Loriot, E. Hertz, O. Faucher, and B. Lavorel, Opt. Express 18, 3011 (2010).

10. J. K. P. Bejot and, S. Henin, V. Loriot, T. Vieillard, E. Hertz, O. Faucher, B. Lavorel, and J.-P. Wolf, Phys. Rev. Lett. 104, 103903 (2010).

11. W. Wang, K. Allaart, and D. Lenstra, Opt. Commun. 278, 395 (2007).

12. M. Kolesik and J. V. Moloney, Phys. Rev. E 70, 036604 (2004).

13. A. Talebpour, J. Yang, and S. L. Chin, Optics Commun. 163, 29 (1999).

14. J. Kasparian, R. Sauerbrey, and S. L. Chin, Appl. Phys. B 71, 877 (2000).

15. F. Théberge, N. Aközbek, W. Liu, J.-F. Gravel, and S. L. Chin, Opt. Commun. 245, 399 (2005).

16. H. Xiong, H. Xu, Y. C. Y. Fu and, Z. Xu, and S. Chin, Phys. Rev. A 77, 043802 (2008).

17. M. Kolesik, E. M. Wright, A. Becker, and J. V. Moloney, Appl. Phys. B 85, 531 (2006). 